\title{
Justice in dealing with highly radioactive waste - an empirical perspective
}

\author{
Lucas Schwarz \\ Environmental Policy Research Centre, Freie Universität Berlin, Berlin, 14195, Germany \\ Correspondence: Lucas Schwarz (lucas.schwarz@fu-berlin.de) \\ Published: 10 November 2021
}

\begin{abstract}
The introduction of the Site Selection Act (Standortauswahlgesetz, StandAG) marked the initiation of a new repository site-selection process as well as the elimination of injustices of past procedures. In this context, the white map of Germany served as a basis for and symbol of an unbiased search process (Hocke and Smeddinck, 2017). However, the publication of the Sub-Areas Interim Report has revealed injustices in the handling of high-level radioactive waste. For example, there is criticism that the methods used to determine the sub-areas are immature or scientifically dubious. There are still fears that rural areas with a low population density will be favored, that there are imbalances in the site-selection process between West and East Germany, or that the discrepancy in the sense of fairness between regions that drop out of the process and those that remain will lead to problems in subsequent stages of the process.

All of these positions show that there is inherent injustice in the search for a repository site: at the end of the site-selection process, a single site will receive all the high-level radioactive waste of the Federal Republic of Germany and thus bear the potential risks. People at this one site will live with the uncertainties associated with dealing with high-level radioactive waste. While the debate about geological or technical factors and challenges is multifaceted and specialized, the issue of fairness in the site-selection process is rarely addressed. However, fairness is immensely important to find a socially acceptable repository site. This paper thus focuses on the following questions: What are the prevailing notions of justice among those involved in the repository siteselection process? From the perspective of the involved parties, what characterizes fairness in the site-selection process?

Answering these questions should contribute to a better understanding of whether the repository site-selection process is perceived as just, and what this depends on. Starting from Rawls' theoretical perspective of justice (2005 [1971]) and Latour's contribution of the values of modern people (2014), an empirical understanding of the stakeholders' perception and understanding of justice in the site-selection process is synthesized. In the context of this work, results of an empirical survey comparing different aspects of justice, e.g., procedural, distributive, intergenerational, and interpersonal, but also justice as recognition, are presented and related to adjacent factors, such as trust, emotions, or experiences. The empirical survey is intended to provide information on whether the perception of justice is more strongly dependent on the process, on one's own affectedness, or on adjacent factors.
\end{abstract}

Kurzfassung. Mit der Einführung des Standortauswahlgesetzes (StandAG) soll der bisherige Standortsuchprozess für ein Endlager neu initiiert und die Ungerechtigkeiten aus vergangenen Verfahren beseitigt werden. Die weiße Landkarte diente dabei als Grundlage und Symbol für einen unvoreingenommenen Suchprozess (Hocke und Smeddinck, 2017). Die Veröffentlichung des Zwischenberichts Teilgebiete hat jedoch offenbart, dass der Umgang mit hochradioaktivem Abfall Ungerechtigkeiten aufweist: So wird kritisiert, dass die Methoden zur Feststellung der Teilgebiete nicht ausgereift bzw. wissenschaftlich zweifelhaft sind, dass es weiterhin die Befürchtung gibt, ländliche Räume mit geringer Bevölkerungsdichte würden bevorzugt, dass Ungleichgewichte zwischen West- und Ostdeutschland im Standortsuchprozess bestehen, oder aber, dass die Diskrepanz des Gerechtigkeitsempfindens zwischen Regionen, die aus dem Verfahren ausscheiden und denen, die im Verfahren bleiben, zu Problemen im späteren Verfahren führen kann. 
All diese Positionen zeigen bereits, dass der Endlagersuche Ungerechtigkeit inhärent ist: Ein einziger Standort wird am Ende des Standortsuchprozesses den gesamten hochradioaktiven Abfall der Bundesrepublik aufnehmen und potenzielle Risiken tragen. Die Menschen an einem Standort werden mit den Unsicherheiten leben, die der Umgang mit hochradioaktivem Abfall mit sich bringt. Während die Debatte über geologische oder technische Faktoren und Herausforderungen bereits vielfältig und fachlich geführt wird, wird das Thema der Gerechtigkeit im Standortsuchprozess kaum behandelt. Es ist aber immens wichtig, wenn das Verfahren erfolgreich sein will, d. h. einen gesellschaftlich akzeptierten Standort für ein Endlager zu finden. Die folgenden Fragen stehen somit im Fokus des Beitrags: Welche Verständnisse von Gerechtigkeit sind bei den Beteiligten im Standortsuchprozess für ein Endlager vorherrschend? Was zeichnet Gerechtigkeit im Standortsuchprozess aus Sicht der Beteiligten aus?

Die Beantwortung der Fragen soll zu einem besseren Verständnis darüber beitragen, ob der Standortsuchprozess für ein Endlager als gerecht empfunden und wahrgenommen wird und wovon dies abhängt. Startend von der theoretischen Perspektive von Rawls zu Gerechtigkeit (2005 [1971]) sowie Latours Beitrag zu den Werten der modernen Menschen (2014), soll ein empirisches Verständnis von Gerechtigkeitsempfinden und -verständnis der Beteiligten im Standortsuchprozess für ein Endlager synthetisiert werden. Dabei sollen im Rahmen des Beitrags Ergebnisse einer empirischen Erhebung vorgestellt werden, die unterschiedliche Aspekte von Gerechtigkeit, zum Beispiel prozedurale, distributive, intergenerationale, zwischenmenschliche, aber auch Gerechtigkeit als Anerkennung miteinander vergleicht und zu angrenzenden Faktoren, wie Vertrauen, Emotionen oder Erfahrungen ins Verhältnis setzt. Durch die empirische Erhebung sollen Aussagen getroffen werden, ob das Gerechtigkeitsempfinden stärker vom Prozess, der eigenen Betroffenheit oder angrenzenden Faktoren abhängig ist.

Note. This contribution can also be presented in English.

Financial support. This research has been supported by the Bundesministerium für Wirtschaft und Energie and the Niedersächsisches Vorab der Volkswagenstiftung (grant no. 02E11849c).

\section{References}

Hocke, P. and Smeddinck, U.: Robust-parlamentarisch oder informell-partizipativ? Die Tücken der Entscheidungsfindung in komplexen Verfahren, GAIA, 26, 125-128, https://doi.org/10.14512/gaia.26.2.16, 2017.

Latour, B.: Existenzweisen: Eine Anthropologie der Modernen, Suhrkamp, Berlin, 2014.

Rawls, J.: A theory of justice, original edn., Belknap Press Harvard University Press, Cambridge, Mass., 607 pp., 2005 [1971]. 